\title{
O USO DA TERMOGRAFIA E CAPTURA DE MOVIMENTOS PARA IDENTIFICAÇÃO DE OPORTUNIDADES DE TECNOLOGIAS ASSISTIVAS: UM ESTUDO DE CASO
}

\section{THE USE OF THERMOGRAPHY AND MOTION CAPTURE TO IDENTIFY ASSISTIVE TECHNOLOGY OPPORTUNITIES: A CASE STUDY}

\author{
Giselle Schmidt Alves Díaz Merino', D.Sc. \\ Irandir Izaquiel Paulo ${ }^{2}$, Bach. \\ Franciele Forcelini ${ }^{3}$, M.Sc. \\ Thiago Varnier ${ }^{4}$, M.Sc. \\ (1) Universidade do Estado de Santa Catarina \\ giselle.merino@gmail.com \\ (2) Universidade Federal de Santa Catarina \\ irandirpitangadesigner@gmail.com \\ (3) Universidade Federal de Santa Catarina \\ francieleforcelini@gmail.com \\ (4) Universidade Federal de Santa Catarina \\ thiagovarnier1@gmail.com
}

Termografia infravermelha, Captura de movimentos, Tecnologia assistiva

Este artigo visa identificar oportunidades para projetos de tecnologias assistivas para um sujeito com deficiência motora a partir da termografia infravermelha e captura de movimentos (Xsens). Os dispositivos oportunos estão relacionados a redução da pressão coxofemoral, proteção dos joelhos, transferência para a cadeira de rodas, personalização do assento, novos revestimentos e propulsão eficiente da cadeira de rodas.

\section{Infrared thermography, Motion capture, Assistive technology}

This article aims to identify opportunities for assistive technology projects for a subject with motor disabilities from infrared thermography and motion capture (Xsens). The appropriate devices are related to the reduction of hip pressure, knee protection, transfer to the wheelchair, personalization of the seat, new coverings and efficient propulsion of the wheelchair.

\section{Introdução}

Tecnologia refere-se ao conhecimento e a aplicação de ferramentas, técnicas e ciências, com o propósito de dar forma ao mundo (BEST, 2012). Engloba o conhecimento, o estudo e o domínio sobre um determinado modo específico de operar ou fazer algo (CIPINIUK; PORTINARI, 2011). As tecnologias impactam nas estruturas de todos os níveis da Gestão de Design, modificando a forma como interagem, viabilizam e comportam novas relações, processos e práticas, proporcionando oportunidades para o design (BEST, 2012).

No processo de design, as tecnologias podem ser incorporadas de diversas maneiras, auxiliando em

\footnotetext{
${ }^{1}$ Equipamentos dotados de distintas tecnologias, os quais são capazes de obter medições precisas e dados quantificáveis.
}

diferentes etapas do projeto. No levantamento de dados, o design pode se utilizar de instrumentos tecnológicos ${ }^{1}$ para a coleta de dados objetivos e quantificáveis, que podem aproximar a equipe de projeto das necessidades reais dos usuários (MERINO et al., 2017; MERINO et al., 2018). Esses instrumentos podem oferecer agilidade ao processo, gerando dados confiáveis que podem tornar os projetos mais eficientes e satisfatórios (FORCELINI; VARNIER; MERINO, 2018). Ou seja, dados quantificáveis e precisos sobre o problema ou necessidade podem minimizar as incertezas do projeto (MERINO et al., 2017; SPECK et al., 2016).

Em coletas com pessoas com deficiência, os instrumentos tecnológicos assumem um papel ainda 
mais significativo, auxiliando na obtenção de dados de sujeitos com diferentes incapacidades (MERINO et al., 2017; SPECK et al.,2016). Possibilitam a obtenção de dados precisos mesmo quando a comunicação com os sujeitos se encontra comprometida (por limitações físicas e psíquicas), impedindo o levantamento de informações por meios como entrevistas, questionários, entre outros (MERINO et al., 2017). A instrumentação permite uma exploração aprofundada dos sujeitos e objetos em estudo, bem como a aproximação entre a equipe de projeto e as capacidades e limitações do usuário, fazendo com que os projetos promovam melhorias na qualidade de vida de pessoas com deficiência ou mobilidade reduzida (FORCELINI; VARNIER; MERINO, 2019; MERINO et al., 2018; VARNIER; MERINO, 2018).

Neste sentido, Merino et al. (2018) destacam que a captura de movimentos (Xsens) e a termografia infravermelha (TIV) são métodos viáveis para o desenvolvimento de tecnologias assistivas (TAs), promovendo soluções mais eficientes, confortáveis e seguras. Essas tecnologias podem auxiliar no desenvolvimento de novos projetos, nas adaptações de produtos existentes, na identificação de problemas e em análises ergonômicas (FORCELINI; VARNIER; MERINO, 2019). Sendo assim, mediante as potencialidades dessas tecnologias, este artigo tem como objetivo identificar oportunidades para projetos de tecnologias assistivas para um sujeito com deficiência motora, a partir da análise de dados quantitativos oriundos da termografia infravermelha e da captura de movimentos (Xsens).

\section{Fundamentação teórica}

\subsection{Termografia Infravermelha}

A termografia infravermelha é um método de detecção da radiação infravermelha emitida pelas superfícies, o qual possibilita o registro das qualidades térmicas de objetos e sujeitos por meio das imagens térmicas geradas (termogramas), que exprimem as variações de temperatura (imperceptíveis a olho nu) por um espectro cromático (CERDEIRA et al, 2011; GABRIEL et al., 2016; MOBLEY, 2002; SILVA, 2017).

Caracteriza-se como um método não destrutivo, sem contato direto, e seus dados podem ser comparados e analisados por meio de softwares específicos, normalmente associados aos fornecedores das câmeras termográficas (GABRIEL et al., 2016;
HOLST, 2000; MOBLEY, 2002; SILVA, 2017).

Destaca-se pela versatilidade, podendo ser utilizada para analisar temperaturas cutâneas (BRIOSCHI; MACEDO; MACEDO, 2003), auxiliar no desenvolvimento e avaliação de produtos (FORCELINI; MERINO, 2019), detectar a deterioração de produtos e defeitos em materiais (SILVA; TARALLI; MELZ, 2015; SILVA; MIRA, 2016), bem como para indicar condições atípicas em qualquer sistema por meio da variação da sua temperatura superficial (CERDEIRA et al., 2011).

Forcelini e Merino (2019) destacam as tecnologias assistivas como a categoria de produtos mais analisada por meio da termografia, demonstrando sua efetividade em projetos envolvendo sujeitos com limitações físicas e psíquicas, devido a sua capacidade de detectar e quantificar temperaturas (dados precisos) de maneira não invasiva e sem interferências. A TIV pode permitir a compreensão do estado muscular do sujeito, podendo auxiliar no desenvolvimento e avaliação de produtos por meio de uma exploração aprofundada e menos subjetiva (MERINO et al., 2018).

\subsection{Captura de movimentos}

A Captura de Movimentos, também chamada de Motion Tracking, trata-se de um método utilizado para mapear os movimentos realizados por um objeto e reproduzi-los em ambiente digital (GOMIDE et al., 2009). Tem a finalidade de registrar o movimento de um corpo por meio de algum dispositivo e, a partir dos dados registrados, calcular as variáveis cinemáticas do movimento (STREIT, 2013).

Dentre os sistemas de captura existentes, o Xsens é um sistema baseado em unidades de medidas inerciais, que combina sinais tridimensionais provenientes de giroscópios e acelerômetros para rastrear o posicionamento do objeto capturado, determinando sua velocidade, aceleração e posição (XSENS, 2012). Neste sistema, os giroscópios são utilizados para medir a orientação dos segmentos corporais e a velocidade de rotação de um objeto em torno de um eixo central. Ou seja, consegue mensurar a velocidade angular, que permite definir o ângulo e orientação dos objetos (CARVALHO, 2011). Os acelerômetros, por sua vez, são dispositivos eletromecânicos que medem a aceleração e a velocidade do objeto, calculando as forças exercidas sobre ele (YANG; HSU, 2010). 
O sistema Xsens capta movimentos em tempo real por meio de 17 sensores inerciais e magnéticos com frequência de $120 \mathrm{~Hz}$ (120 frames por segundo), o que possibilita a avaliação contínua do movimento dinâmico (XSENS, 2012). Logo, os dados obtidos são transmitidos por conexão wireless para um computador e, por meio de um software específico, podem ser observados, gravados e analisados com o auxílio de gráficos dos ângulos das articulações, da velocidade e duração dos movimentos (SPECK et al., 2016; ROETENBERG; LUINGE; SLYCKE, 2013).

Varnier e Merino (2018) apontam que a captura de movimentos facilita o levantamento de informações precisas e quantificáveis dos movimentos de segmentos e articulações, tornando-se um complemento positivo para as medidas subjetivas. Desta forma, sua utilização possibilita a criação de novos produtos e auxilia na identificação dos requisitos de projeto, podendo ser uma ferramenta eficiente para a obtenção de dados quantitativos na área de design, gerando informações e definições precisas da biomecânica (VARNIER; MERINO, 2018).

\subsection{Tecnologia Assistiva (TA)}

No mundo, um em cada sete indivíduos possuem algum tipo de deficiência (motora, sensorial e/ou cognitiva) e, no Brasil, isso corresponde a 45 milhões de indivíduos (BRASIL, 2012; WHO, 2012). Portanto, o uso e o desenvolvimento de tecnologias assistivas vem se tornando cada vez mais necessário.

TAs são produtos, recursos, estratégias, práticas, processos, métodos e serviços com a finalidade de promover a funcionalidade de indivíduos com deficiência ou mobilidade reduzida, visando sua autonomia, independência, qualidade de vida e inclusão social (BRASIL, 2015). Caracterizam-se como um subsídio na melhoria das capacidades funcionais negativas dos indivíduos, que possibilita a realização da atividade desejada, antes bloqueada por algum tipo de deficiência ou envelhecimento (BERSCH, 2017).

No que se refere ao desenvolvimento de projetos de TAs, a atuação do designer pode possibilitar produtos e/ou serviços favoráveis ao ambiente no qual estão inseridos, facilitado a inclusão das pessoas com deficiência (BASSO, 2012). Para o desenvolvimento de projetos, o usuário deve ser totalmente incluído, considerando-se seu contexto de vida, suas capacidades funcionais e habilidades (BERSCH, 2017). Esse conhecimento sobre o potencial motor, sensorial e cognitivo do usuário possibilita a avaliação de TAs existentes e o desenvolvimento de novos projetos para uma necessidade específica (BERSCH, 2017). Assim, os dispositivos assistivos podem suprir uma capacidade comprometida, diminuir as implicações de uma alteração gradativa, reduzir a carência de cuidadores, bem como evitar ocorrências de outras enfermidades (WHO, 2012).

\section{Procedimentos Metodológicos}

O presente estudo possui natureza aplicada, utilizando seus resultados para a solução de problemas reais (MARCONI; LAKATOS, 2007). Sua abordagem é qualitativa, uma tentativa de compreender detalhadamente características situacionais (MARCONI; LAKATOS, 2007) do sujeito da pesquisa - cadeirante com paraplegia e atrofias nos membros superiores e inferiores.

Quanto aos objetivos, classifica-se como exploratório, descritivo e correlacional. É exploratório pois visa examinar um problema pouco estudado, familiarizando-se com fenômenos desconhecidos, contextos específicos e novos problemas (SAMPIERI; COLLADO; LUCIO, 2013). É descritivo pois busca especificar as características de um fenômeno para sua análise, coletando informações sobre suas variáveis, indicando como estas se relacionam (SAMPIERI; COLLADO; LUCIO, 2013). E também é correlacional, visto que visa conhecer a relações existentes entre as variáveis de um contexto específico (SAMPIERI; COLLADO; LUCIO, 2013). Ou seja, neste estudo exploram-se as condições específicas de um sujeito com deficiência física, visando a descrição das suas condições (com base em dados gerados pelo uso da instrumentação tecnológica) e a correlação dos dados para identificar oportunidades de projeto.

Sendo assim, este estudo segmenta-se em três etapas: (1) Levantamento de dados; (2) Analise de dados e; (3) Identificação de oportunidades de novos projetos.

- Levantamento de dados - contemplou o levantamento de dados por meio da termografia infravermelha e captura de 
movimentos, registros fotográficos, de vídeo e observações assistemáticas.

- Analise de dados - contemplou a descrição e análise dos dados levantados

- Identificação de oportunidades de novos projetos - foram identificadas novas oportunidades de projetos de TAs para o sujeito analisado.

\subsection{Materiais e métodos}

A etapa 1 - Levantamento de dados foi realizada com um sujeito com deficiência (paraplegia, encurtamento dos tendões e sensibilidade do lado esquerdo do corpo), do sexo feminino, com 24 anos de idade, usuário de cadeira de rodas.

O sujeito foi informado sobre o objetivo do estudo, consentindo com a sua realização por meio da assinatura do Termo de Consentimento Livre e Esclarecido (TCLE). Além disso, por se tratar de um estudo envolvendo um ser humano, esse estudo encontra-se associado a um projeto aprovado pelo Comitê de Ética em Pesquisa com Seres Humanos (CEPSH) da Universidade Federal de Santa Catarina $\left(n^{\circ} 2.732 .152\right)$.

As coletas foram realizadas na Universidade Federal da Paraíba (UFPB), no campus de Rio Tinto e, para tanto, foram utilizados procedimentos específicos para cada método - termografia infravermelha e captura de movimentos.

Para a realização da coleta com a termografia infravermelha foi utilizada uma versão preliminar do protocolo Thermos, que tem o objetivo de guiar o processo da coleta termográfica, manter seu rigor e confiabilidade dos dados obtidos (FORCELINI, 2019; FORCELINI; MERINO, 2019). Com suas orientações, foram registrados os dados sobre: (1) o contexto de coleta (layout do ambiente, variáveis ambientais e equipamentos utilizados); (2) o usuário (sujeito dessa pesquisa) e; (3) o produto analisado (cadeira de rodas).

No que se refere ao contexto da coleta, o espaço foi organizado conforme os layouts apresentados na Figura 1, considerando o posicionamento do base negra em TNT (01), do tripé com a câmera termográfica (02), da usuária/sujeito da pesquisa (03) e do produto/cadeira de rodas (04).

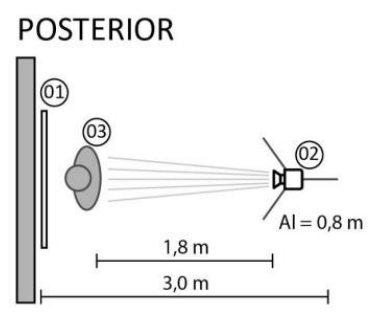

CADEIRA DE RODAS

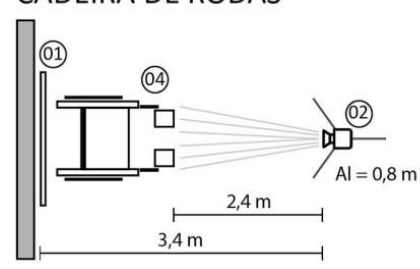

Figura 1 - Layout da coleta de dados termográficos. Fonte: os autores.

As variáveis ambientais foram controladas durante toda a coleta, mantendo-se a temperatura em $25^{\circ} \mathrm{C}$, a umidade do ar em $55 \%$ e a velocidade do ar em $0 \mathrm{~m} / \mathrm{s}$.. Para as medições ambientais foi utilizado o Termo-Higro-Anemômetro THAL 300, da Instrutherm e, para o registro dos dados termográficos, o termovisor FLIR E40 (com calibração automática).

Quanto o sujeito, foram definidas três áreas para análise - mãos (1), costas (2) e membros inferiores (3) -, as quais passaram por um período de aclimatização de 15 minutos, conforme sugere Alves Neto et al. (2009). Após a aclimatização, foi realizado o primeiro registro de cada região, em repouso (TR), e o segundo imediatamente após a realização da atividade proposta (T0). Para o registro das mão e costas, o sujeito foi orientado a deslocarse utilizando a cadeira de rodas por um trajeto de 10 metros, sem desníveis (duração aproximada de 4 minutos). Já para o registro da região dos membros inferiores (T0), o sujeito foi orientado a deslocar-se por cerca de 5 metros sem o uso da cadeira de rodas (imagem 2), do modo com que costuma deslocar-se diariamente. Essa distância limitada foi determinada em função da sua dificuldade de deslocamento. Além destes, também foi realizado o registro da cadeira de rodas utilizada pelo sujeito (posição frontal).

Para a captura de movimentos, foi utilizado uma versão prévia do protocolo Motion Capture (VARNIER, 2019; VARNIER; MERINO, 2019), que propõem um conjunto de orientações para guiar as equipes de projeto no levantamento de dados com os usuários por meio do equipamento MVN Biomech, da Xsens. Este sistema é baseado em 
PUC-Rio Pontifícia Universidade Católica do Rio de Janeiro Departamento de Artes \& Design | PPGDesign

LEUI | Laboratório de Ergodesign e Usabilidade de Interfaces

unidades de medida inercial, utilizando 17 sensores que permitem registar o movimento $3 \mathrm{D}$ de 23 segmentos corporais e 22 articulações, bem como o centro de massa, em tempo real (GOMIDE et al.,2009; ROETENBERG; LUINGE; SLYCKE, 2013; XSENS, 2012).

A captura dos movimentos foi realizada com isolamento dos membros inferiores, utilizando apenas os sensores inerciais nos membros superiores (cabeça, tronco, pélvis e braços). Dessa forma, realizou-se: (1) a mensuração das medidas do corpo; (2) a fixação dos sensores inerciais ao corpo e a ligação dos fios de captura; (3) a calibração do equipamento, seguindo a pose "Npose" - posição neutra; (4) a gravação dos movimentos e, por fim; (5) a análise dos dados cinemáticos no software.

As atividades gravadas foram: (1) transferência para cadeira de rodas; (2) transferência da cadeira de rodas para o chão; (3) girar no chão para ficar de joelhos (posição de marcha do sujeito) e; (4) caminhar de joelhos (marcha padrão do sujeito). Os tempos de gravação da captura de movimento variaram de 30 segundos a 1 minuto.

Além das coletas com os instrumentos tecnológicos, também foram feitos registros fotográficos, de vídeo e observações assistemáticas. As observações assistemáticas qualitativas aconteceram por meio de um papel ativo e uma reflexão constante sobre as situações, com atenção aos detalhes, acontecimentos e interações do sujeito (SAMPIERI; COLLADO; LUCIO, 2013). Todas as análises foram realizadas por uma equipe multidisciplinar, que incluiu designers e fisioterapeutas.

Na etapa 2 - Análise dos dados, os dados termográficos foram extraídos por meio do software Flir Tools e organizados em planilhas do Microsoft Excel para a identificação das temperaturas dos pontos hiper radiantes (com temperatura mais elevada), as médias e as diferenças das temperaturas nos diferentes tempos. Os parâmetros adotados para a padronização das imagens termográficas são apresentados na Tabela 1.

\begin{tabular}{|l|l|}
\hline Emissividade & $\begin{array}{l}0,98 \text { - Padrão para medições de pele } \\
\text { (GABRIEL et al., 2016). }\end{array}$ \\
\hline Temp. Refl. & $20^{\circ} \mathrm{C}$ \\
\hline Distâncias & Variadas (Figura 1) \\
\hline Temp. atmosférica & $25^{\circ} \mathrm{C}$ \\
\hline Temp. da óptica ext. & ${ }^{\circ} \mathrm{C}$ \\
\hline Trans. da óptica ext. & 1 \\
\hline Umidade relativa & $55 \%$ \\
\hline
\end{tabular}

Tabela 1 - Parâmetros para padronização das imagens termográficas. Fonte: os autores.
Os dados da captura de movimentos foram extraídos do software do equipamento MVN Biomech para uma planilha do Microsoft Excel, possibilitando a análise cinemática quanto a posição (ângulos).

\section{Na etapa 3 - Identificação de oportunidades de} novos projetos, com base na análise dos dados foram identificadas oportunidades de novos projetos de TAs para o sujeito em questão.

\section{Resultados e discussões}

Mediante as observações foi possível o entendimento da rotina do sujeito da pesquisa, que foi caracterizado como semi-dependente, visto que realiza algumas atividades de forma autônoma e outras com auxílio. Para sentar, o sujeito assume uma postura em w, conforme demonstra a Figura 2A. Para caminhar (Figura 2-B) em sua residência e áreas próximas, mantém-se ajoelhado (em contato direto com diferentes superfícies) e, para locomoverse em ambientes externos, utiliza uma cadeira de rodas (Figura 3-A).

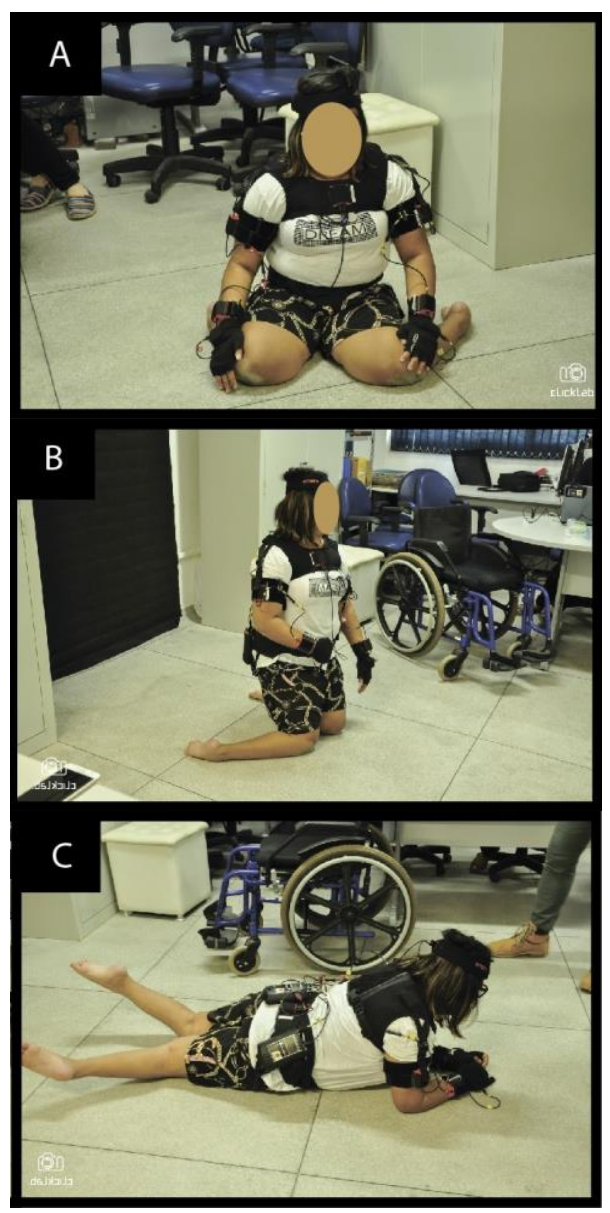

Figura 2 - Sujeito da pesquisa (marcha e uso da cadeira de roda). Fonte: os autores. 
O sujeito também possui dificuldade na transferência do chão para a cadeira e vice-versa, necessitando do auxílio de outras pessoas, conforme pode ser observado na Figura 3 (A, B e C).

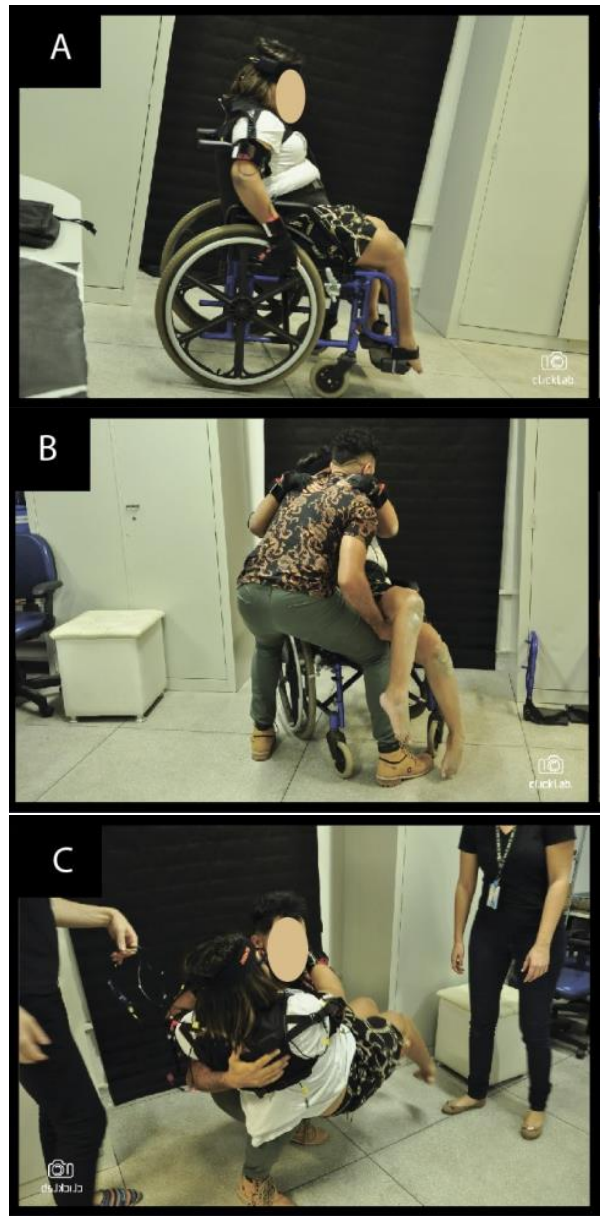

Figura 3 - Sujeito da pesquisa (transferência). Fonte: os autores.

Durante sua transferência, o sujeito é colocado no chão para poder ficar na posição de andar (Figura 2C), que o pode gerar constrangimento (pelo contato com superfícies desconfortáveis e sujas) e risco de lesões. Deste modo, destaca-se a oportunidade de desenvolvimento de um dispositivo que permita a transferência do sujeito de forma autônoma e segura, eliminando a necessidade de auxílio de outras pessoas.

Com observação dos vídeos e imagens da marcha e da posição de repouso, foi verificado importante uso de rotação interna bilateral dos quadris, que é um fator de risco para o desenvolvimento de artrose e lesões labrais devido a acentuação do processo degenerativo articular. Este risco foi corroborado pela avaliação termográfica da região posterior do sujeito, que apresentou um aumento na temperatura das articulações coxofemorais (Figura 4 e 5).
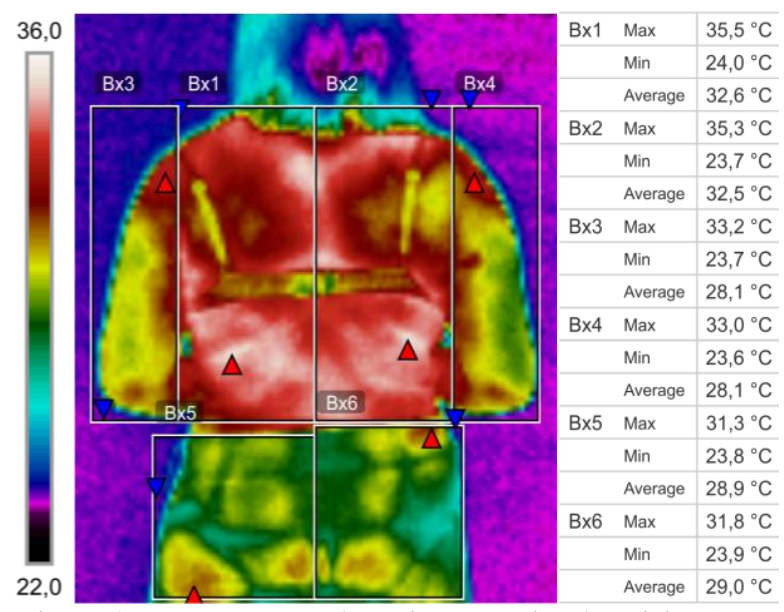

Figura 4 - Termogramas da região posterior do sujeito (TR). Fonte: os autores.

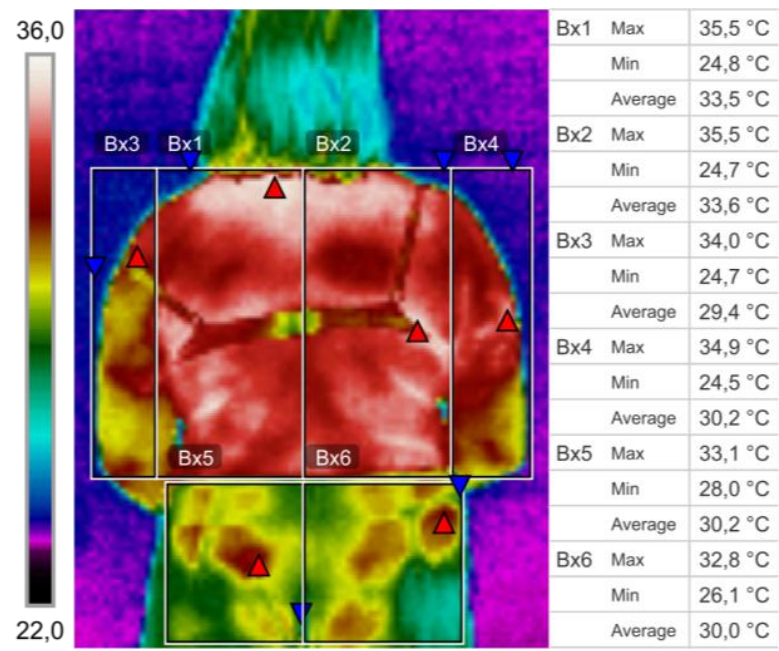

Figura 5 - Termogramas da região posterior do sujeito (T0). Fonte: os autores.

Após deslocamento com a cadeira de rodas, a região dos glúteos apresentou pontos hiper radiantes (ponto com temperatura mais elevada) $\operatorname{com} 33,1^{\circ} \mathrm{C}$ (lado esquerdo) e $32,8^{\circ} \mathrm{C}$ (lado direito). Essas temperaturas demonstratam um aumento significativo considerando que a temperatura média em repouso foi de $23,8^{\circ} \mathrm{C}$ (lado esquerdo) e de $23,9^{\circ} \mathrm{C}$ (lado direito). Esses dados termográficos demonstram o grau de vasoconstrição ou de vasodilatação da pele, que pode refletir o funcionamento, disfunção ou respostas a um traumatismo (BRIOSCHI et al., 2009). Não obstante, pressão excessiva aplicada no tecido também pode gerar mudanças térmicas (BRIOSCHI et al., 2009).

Assim, propõe-se o desenvolvimento de um dispositivo assistivo para redução da pressão coxofemorais observada, que possibilite a 
PUC-Rio Pontifícia Universidade Católica do Rio de Janeiro

\section{estabilização e a mobilidade do sujeito de} maneira confortável e segura.

Os dados termográficos da região posterior após deslocamento com a cadeira de rodas (Figura 5) também demonstraram uma elevação nas temperaturas do braço direito (deltoide) e dos trapézios (bilateralmente). $\mathrm{O}$ braço direito apresentou um aquecimento de $1,9^{\circ} \mathrm{C}$ no ponto hiper radiante $\left(33^{\circ} \mathrm{C}\right.$ para $\left.34,9^{\circ} \mathrm{C}\right)$, o que pode estar relacionado a assimetria da força aplicada para a propulsão da cadeira de rodas, provocada por um visível desvio na coluna do sujeito. Neste contexto, tem-se como oportunidade de projeto o desenvolvimento de um sistema de propulsão que reduza a aplicação de força para a movimentação da cadeira de rodas.

Ainda, os termogramas ilustraram o aumento das temperaturas dos trapézios bilateralmente, o que pode estar relacionado a protrusão (anteriorização) da cabeça, demonstrada pela captura de movimentos das articulações T1-C7 e C1-Read (Figura 6).

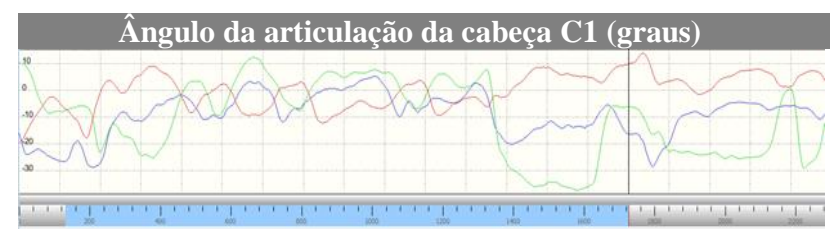

Flexão lateral direita(+) e esquerda(-) Flexão(+)/Extensão(-) Rotação axial

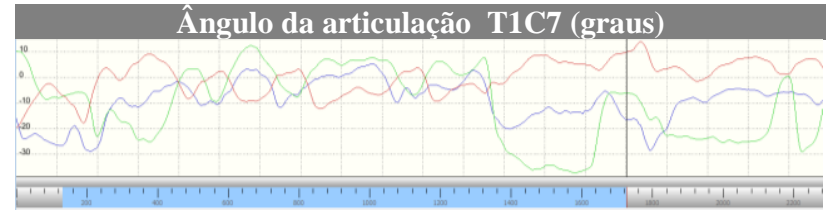

Figura 6 - Captura de movimentos do sujeito durante a marcha. Fonte: os autores.

A análise dessas articulações durante o caminhar de joelhos (marcha padrão do sujeito) demonstrou a protrusão da cabeça com ângulos de extensão entre $20^{\circ}$ a $30^{\circ}$, acima da zona de conforto estabelecida entre $0^{\circ}$ e $15^{\circ}$ (TILLEY; DREYFUSS, 2005). A manutenção dessa postura anteriorizada da cabeça resulta no aumento das cargas compressivas sobre a coluna cervical e pode causar a deformação dos tecidos (BONNEY; CORLETT, 2002).

O aquecimento dos trapézios e do deltoide direito, podem estar associados aos movimentos de flexão e abdução dos ombros (Figura 7). A amplitude do movimento do ombro apresentou ângulos de extensão acima de $60^{\circ}$, acima do intervalo de conforto de $0^{\circ}$ a $45^{\circ}$ (PALMER; EPLER, 2000; MARQUES, 2003). Assim, reforça-se a necessidade de um dispositivo que possa auxiliar na estabilização e mobilidade do sujeito, reduzindo a extensão dos ombros e as lesões nos joelhos e nas articulações T1-C7, C1- Head.

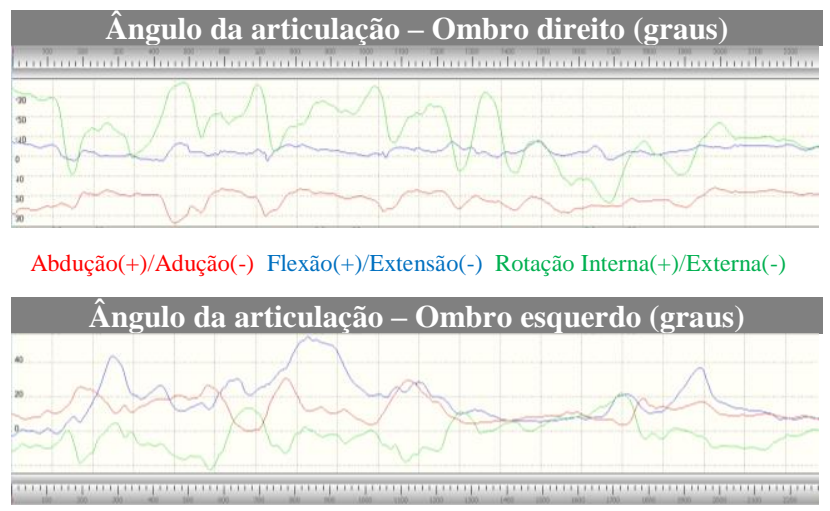

Figura 7 - Captura de movimentos dos ombros (dir. e esq.) Fonte: os autores.

Os dados termográficos das mãos (Figura 8 e 9) demonstraram o aumento das temperaturas após a propulsão da cadeira de rodas, $1,1^{\circ} \mathrm{C}$ na mão direita e a $1,7^{\circ} \mathrm{C}$ na mão esquerda. Este aquecimento pode estar relacionado à pressão excessiva aplicada no tecido durante a propulsão da cadeira, o quak pode gerar mudanças térmicas (BRIOSCHI et al., 2009).
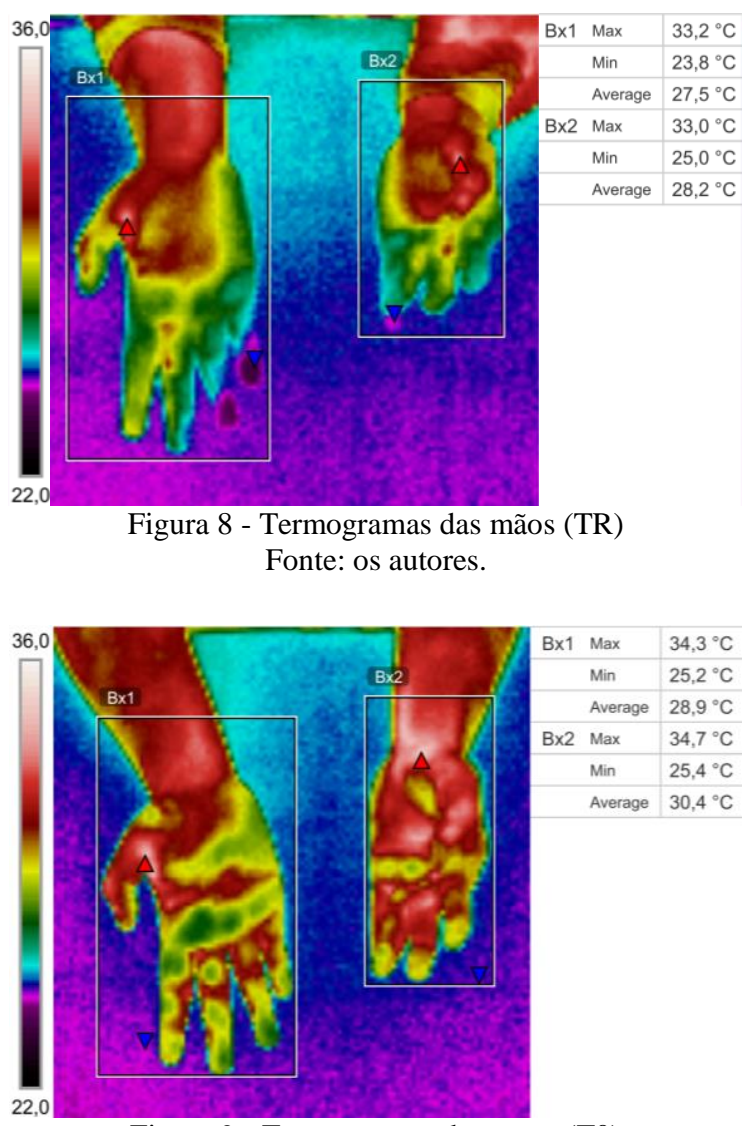

Figura 9 - Termogramas das mãos (T0) Fonte: os autores. 
O ponto hiper radiante da mão direita foi encontrado na região tenar (em repouso e após a atividade), e na esquerda passou da região tenar (em repouso) para a articulação localizada entre a mão e o punho (após atividade). Isso pode estar associado à atrofia existente na mão esquerda, que dificulta o manuseio do sistema de propulsão. Assim, para reduzir a pressão sobre a pele e as articulações, destaca-se a oportunidade para o desenvolvimento de um novo sistema de propulsão para a cadeira de rodas, que reduza a necessidade de força, facilitando seu manuseio e diminuindo o atrito com as mãos.

Mediante os dados termográficos dos membros inferiores do sujeito (Figura 10 e 11) foram observadas temperaturas mais baixas na região das pernas, o que pode ser associado a menor circulação sanguínea provocada pelas atrofias musculares. Percebe-se uma assimetria nos joelhos, com maior temperatura no joelho direito. Além disso, foi observada a redução da temperatura das pernas após deslocamento em contato com o piso frio do local. A média de temperatura da perna direita reduziu $0,9^{\circ} \mathrm{C}$ e da perna esquerda $1,2^{\circ} \mathrm{C}$.

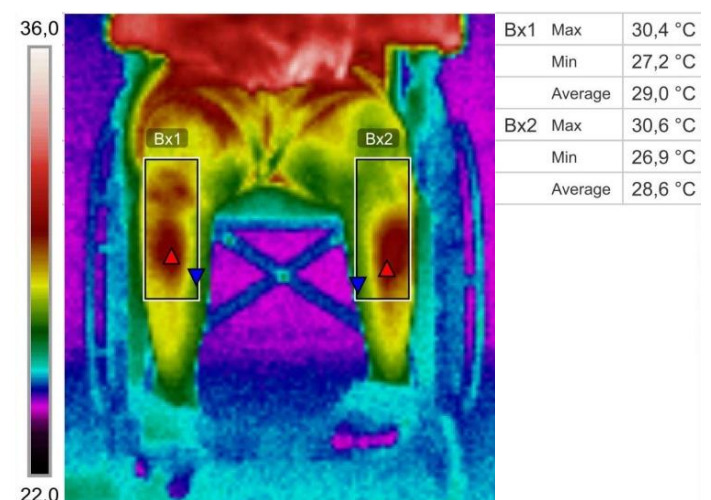

Figura 10 - Termogramas dos membros inferiores (TR) Fonte: os autores.

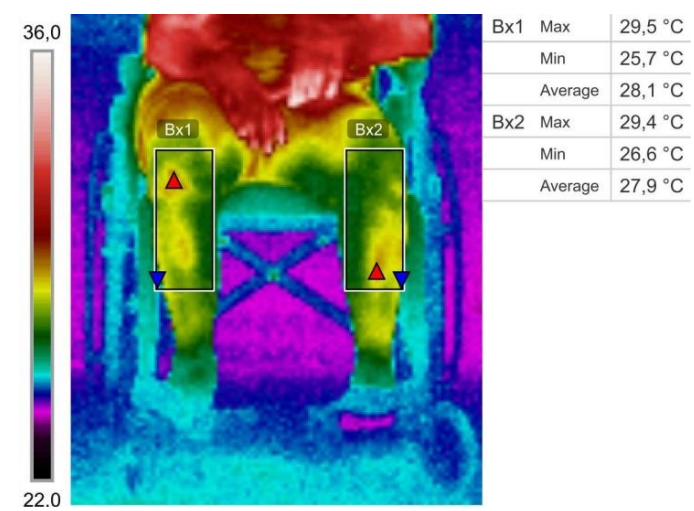

Figura 11 - Termogramas dos membros inferiores (T0) Fonte: os autores.
Com bases nestas medições foi possível identificar as regiões de contato com o piso, as quais podem orientar o desenvolvimento de um dispositivo para proteção de joelhos e demais regiões que possuem contato com o chão. Essa solução poderia ser combinada com o dispositivo para estabilização e mobilidade, proposto anteriormente, permitindo seu uso combinado ou independente. Esse dispositivo poderia apresentar superfície com materiais confortáveis internamente e resistentes externamente.

Por fim, os dados termográficos da cadeira de rodas (Figura 12 e 13) demonstraram a elevação nas temperaturas do assento $\left(4^{\circ} \mathrm{C}\right)$ e do encosto $\left(2,9^{\circ} \mathrm{C}\right)$ após o deslocamento. Mediante este aquecimento, percebe-se a oportunidade de desenvolver e testar diferentes revestimento para a cadeira de rodas, os quais possam reduzir seu aquecimento e melhorar o conforto térmico da usuária.

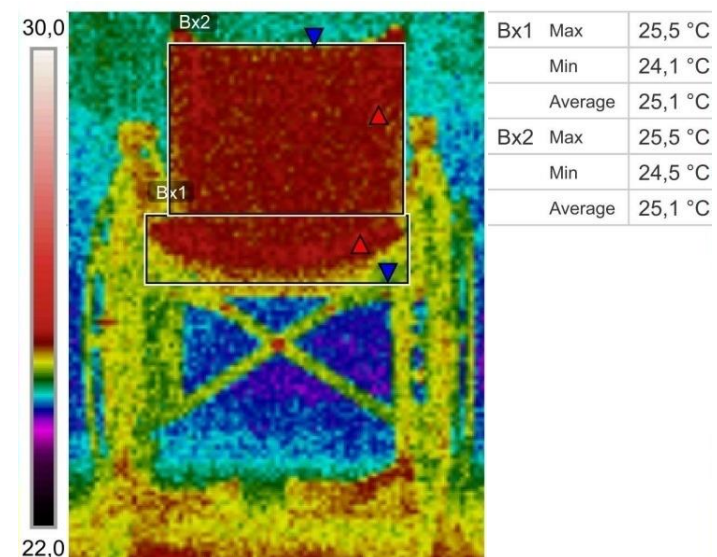
Figura 12 - Termogramas da cadeira de rodas (TR). Fonte: os autores.

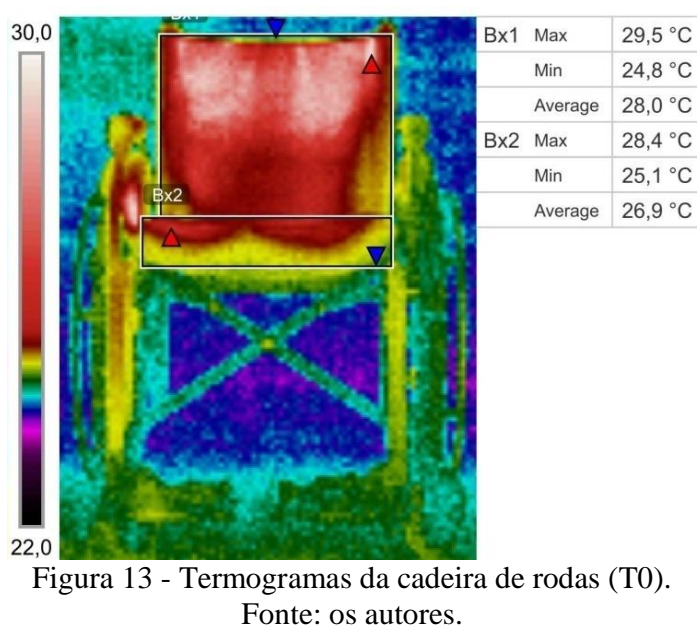

O termograma da cadeira de ropas, após seu uso, demonstrou uma assimetria nas temperaturas do assento, associada ao comprometimento físico da 
usuária. Nesse sentido, identifica-se a possibilidade de desenvolver um assento e/ou um encosto personalizado, com base em dados antropométricos da usuária da cadeira de rodas, que tornaria homogênea a área de contato com o corpo, aliviando as pressões sobre a pele.

\section{Conclusão}

Este estudo demonstrou condições problemáticas relacionadas às posturas do sujeito e ao seu deslocamento, com e sem o uso da cadeira de rodas.

Com base nas análises realizadas por profissionais da área de design e da saúde, foi possível a identificação de oportunidades de projeto, como: (1) desenvolvimento de um dispositivo assistivo para estabilização e mobilidade, que permita a redução da pressão coxofemorais; (2) desenvolvimento de um novo sistema de propulsão para a cadeira de rodas, que reduza a necessidade de força, facilitando o manuseio e diminuindo o atrito com as mãos da usuária; (3) desenvolvimento de um dispositivo para proteção de joelhos e demais regiões de contato com o chão durante a marcha; (4) mudança do revestimento da cadeira de rodas, com o propósito de reduzir o aquecimento e melhorar o conforto térmico da usuária; (5) desenvolvimento de um assento e/ou encosto personalizado, com base em dados antropométricos da usuária da cadeira de rodas e; (6) desenvolvimento de um dispositivo que permita a transferência de forma autônoma e segura.

De modo geral, essas oportunidades poderiam auxiliar o sujeito na realização de suas atividades da vida diária, aumentando seu conforto e segurança, reduzindo o risco de lesões e melhorando sua qualidade de vida. No entanto, para desenvolvê-las, salienta-se a necessidade de uma análise detalhada e multidisciplinar do sujeito (fisioterapeutas, médicos, terapeutas ocupacionais, designers, etc.).

Em relação aos procedimentos adotados, as observações contribuíram para visualizar as interações da usuária em diferentes contextos, auxiliando na identificação das suas dificuldades. A termografia infravermelha se mostrou eficiente para a coleta de dados com um sujeito com deficiência, destacando-se por seu caráter não invasivo, que permite análises sem a necessidade de contato direto. A captura de movimentos, por sua vez, se mostrou adequada para a simulação real dos movimentos, permitindo definições que contribuíram para o entendimento das necessidades do sujeito. Os profissionais da saúde foram essenciais para a análise dos dados.

Como estudos futuros sugere-se a elaboração de pesquisas focadas em cada uma das oportunidades. Estas pesquisas teriam como o objetivo de levantar dados específicos sobre cada problema, apresentando requisitos para o desenvolvimento dos projetos, alternativas, estudos de materiais, etc.

\section{Referências Bibliográficas}

ALVES NETO, O. et al. Dor: princípios e prática. Porto Alegre: Artmed, 2009.

\section{BASSO, Liliane. A contribuição do designer no} projeto de recursos de tecnologia assistiva: proposta de intervenção colaborativa. 2012. $175 \mathrm{f}$. Dissertação (Mestrado) - Curso de Design, Universidade Federal do Rio Grande do Sul, Porto Alegre, 2012. Disponível em:

<https://www.lume.ufrgs.br/bitstream/handle/10183/ 62048/000867041.pdf? sequence=1\&isAllowed $=\mathrm{y}>$. Acesso em: 02 set. 2019.

BERSCH, R. Introdução à Tecnologia Assistiva. Porto Alegre: Assistiva - Tecnologia e Educação, 2017.

BEST, Kathryn. Fundamentos da gestão do design. Porto Alegre: Bookman, 2012.

BONNEY, Rosemary A.; CORLETT, Esmond Nigel. Head posture and loading of the cervical spine. Applied ergonomics, v. 33, n. 5, p. 415-417, 2002.

BRASIL. Cartilha do Censo 2010: Pessoas com deficiência. Secretária de Direitos Humanos da Presidência da República (SDH-PR); Secretaria Nacional dos Direitos da Pessoa com Deficiência (SNPD), et al. Brasilia: SDH-PR/SNPD, 2012.

BRASIL. Lei 13.146 de 06 de julho de 2015. Institui a Lei Brasileira de Inclusão da Pessoa com Deficiência (Estatuto da Pessoa com Deficiência). Diário Oficial da União, 2015.

BRIOSCHI, Marcos L.; MACEDO, José F; MACEDO, Rodrigo de A. C. Termometria cutânea: novos conceitos. Jornal Vascular Brasileiro, v. 2, p. 151-60, 2003. 
PUC-Rio Pontifícia Universidade Católica do Rio de Janeiro Departamento de Artes \& Design | PPGDesign

BRIOSCHI et al. Termografia no diagnóstico da dor. In: ALVES NETO, O. et al. Dor: princípios e prática. Porto Alegre: Artmed, 2009.

CARVALHO, Adriano Guedes. Influência da modelagem dos componentes de bias instabilidade dos sensores inerciais no desempenho do navegador integrado SNI/GPS. 2011. 146f. Dissertação (Mestrado) - Curso de Pósgraduação em Engenharia Elétrica. Instituto Militar de Engenharia, Rio de Janeiro, 2011. Disponível em: < http://pgee.ime.eb.br/pdf/adriano_carvalho.pdf> Acesso em: 23 ago. 2019.

CERDEIRA, Fernando et al. Applicability of infrared thermography to the study of the behaviour of stone panels as building envelopes. Energy and Buildings, v. 43, n. 8, p. 1845-1851, 2011.

FORCELINI, Franciele; MERINO, Eugenio Andrés Díaz. Termografia Infravermelha no

Desenvolvimento de Produtos: formas de abordagem e categorias dos produtos, p. 2665-2678. In: Anais do $13^{\circ}$ Congresso Pesquisa e Desenvolvimento em Design (2018). São Paulo: Blucher, 2019. Disponível em:

<https://www.proceedings.blucher.com.br/articledetails/termografia-infravermelha-nodesenvolvimento-de-produtos-formas-deabordagem-e-categorias-dos-produtos-30139>. Acesso em: 20 ago. 2019.

FORCELINI, Franciele; MERINO, Giselle Schmidt Alves Díaz. Guia de Orientações Thermos Protocol. Florianópolis: Ngd/Ufsc, 2019. Disponível em: <www.ngd.ufsc.br/thermosprotocol/>. Acesso em: 13 ago. 2019.

\section{FORCELINI, Franciele. Termografia} infravermelha aplicada ao design: protocolo de coleta de dados termográficos para o desenvolvimento de projetos. Dissertação (Mestrado em Design) - Programa de Pós-Graduação em Design, Universidade Federal de Santa Catarina. Florianópolis, 247 p., 2019.

FORCELINI, Franciele; VARNIER, Thiago; MERINO, Eugenio Andrés Díaz. Termografia Infravermelha e Captura de Movimentos: vantagens e desvantagens no desenvolvimento de projetos, $\mathrm{p}$. 2652-2664. In: Anais do $\mathbf{1 3}^{\circ}$ Congresso Pesquisa e Desenvolvimento em Design (2018). São Paulo: Blucher, 2019. Disponível em: $<$ https://www.proceedings.blucher.com.br/article- details/termografia-infravermelha-e-captura-demovimentos-vantagens-e-desvantagens-nodesenvolvimento-de-projetos-30138>. Acesso em: 20 ago. 2019.

GABRIEL, Joaquim et al. Termografia: imagem médica e síndromes dolorosas. Lisboa: Lidel, 2016.

GOMIDE, João Victor Boechat et al. Captura de movimento e Animação de Personagens em Jogos. In: Brazilian Symposium on Games and Digital Entertainment, 8., 2009, Rio de Janeiro. Simpósio... Rio de Janeiro: PUC, 2009.

HOLST, Gerald C. Common sense approach to thermal imaging. Washington, DC, USA: SPIE Optical Engineering Press, 2000.

MARCONI, Marina de Andrade; LAKATOS, Eva Maria. Técnicas de pesquisa: planejamento e execução de pesquisas, amostragens e técnicas de pesquisas, elaboração, análise e interpretação de dados. 6.ed. São Paulo: Atlas, 2007.

MARQUES A. P. Ângulos articulares dos membros superiores. In: Manual de Goniometria. 2 ed. São Paulo: Editora Manole. 2003, p.12-17.

MERINO, Eugenio A. D. et al. Implementation of Integrated Instrumentation in Assistive Technology. Advances In Ergonomics In Design, [s.1.], p.549560, 24 jun. 2017. Springer International Publishing. http://dx.doi.org/10.1007/978-3-319-60582-1_55.

MERINO, Eugenio A. D. et al. O uso da instrumentação tecnológica em projetos de tecnologia assistiva: captura de movimentos e termografia infravermelha. Human Factors In Design, [s.1.], v. 7, n. 14, p.95-113, 15 out. 2018. Universidade do Estado de Santa Catarina. http://dx.doi.org/10.5965/2316796307142018095.

MOBLEY, R. Keith. An introduction to predictive maintenance. 1. ed. Amsterdam: ButterworthHeinemann, 2002.

PALMER, Lynn M.; EPLER, Marcia E. Ombro. In: Fundamentos das Técnicas de Avaliação Musculoesquelética. 2. ed. Rio de Janeiro: Guanabara Koogan; 2000. p.63-108.

ROETENBERG, Daniel; LUINGE, Henk; SLYCKE, Per. Xsens MVN: full 6DOF human 
motion tracking using miniature inertial sensors.

Xsens Motion Technologies BV, v.3, p. 1-9, 2013.

SAMPIERI, Roberto H.; COLLADO, Carlos F.; LUCIO, M. P. B. Métodos de Pesquisa. 5. ed. Porto Alegre: Penso, 2013.

SILVA, Júlio César R. P.; MIRA, Maria do R. G. Termografia: ferramenta auxiliar na pesquisa de materiais e no design de produtos. Libro de Actas Systems \& Design: Beyond Processes and Thinking (IFDP - SD2016), [s.1.], p.377-391, 22 jun. 2016. Universitat Politècnica València. http://dx.doi.org/10.4995/ifdp.2016.3651.

Disponível em: . Acesso em: 10 jun. 2017.

SILVA, Júlio Cézar R. P. da; TARALLI, Cibele H.; MELZ, Simone P. M. Termograma: A imagem térmica como instrumento de diagnóstico rápido no design. In: Fourth International Conference on Integration of Design, Engineering and Management for innovation. Anais... Florianópolis, SC, Brasil: 2015. Disponível em: <http://janainaramos.com.br/idemi2015/anais/02/14 3332.pdf>. Acesso em: 14 set. 2017.

SILVA, Luiz A. da. Termografia: Princípios básicos e suas aplicações. 1. ed. São José dos Campos: Tecnolass Tecnologia, 2017.

SPECK, Giselle Mari et al. Processo de instrumentação integrada no desenvolvimento de projetos de Tecnologia Assistiva. Anais de congresso $18^{\circ}$ Congresso Brasileiro de Ergonomia (ABERGO), Belo Horizonte: 2016.

\section{TILLEY, A. R.; HENRY DREYFUSS}

ASSOCIATES. As medidas do homem e da mulher: fatores humanos em design. Porto Alegre: Bookman, 2005.

VARNIER, Thiago. Fatores Humanos associados aos projetos de Design: protocolo de coleta para a captura de movimentos. Dissertação (Mestrado em Design) - Programa de Pós-graduação em Design, Universidade Federal de Santa Catarina, Florianópolis, 250 p., 2019.

VARNIER, Thiago; MERINO, Eugenio Andrés Díaz. O Uso da Captura de Movimentos no Desenvolvimento de Produtos: um estudo focado nas tecnologias e aplicações, p. 2463-2477. In: Anais do $13^{\circ}$ Congresso Pesquisa e Desenvolvimento em Design (2018). São Paulo:
Blucher, 2019. Disponível em:

<https://www.proceedings.blucher.com.br/articledetails/o-uso-da-captura-de-movimentos-nodesenvolvimento-de-produtos-um-estudo-focadonas-tecnologias-e-aplicaes-30125>. Acesso em: 20 ago. 2019.

VARNIER, Thiago; MERINO, Giselle Schmidt Alves Díaz. Motion Capture Protocol: Protocolo de coleta MVN Link Biomech. Florianópolis: NGD/UFSC, 2019. Disponível em:

〈www.ngd.ufsc.br〉. Acesso em: 16 ago. 2019.

WHO. Relatório mundial sobre a deficiência. SEDPcD. São Paulo, p.334. 2012.

XSENS. Moven: user manual. Moven Motion Capture System. The Netherlands: Xsens Technologies B.V. 2012.

YANG, Che-Chang; HSU, Yeh-Liang. A review of accelerometry-based wearable motion detectors for physical activity monitoring. Sensors, v. 10, n. 8, p. 7772-7788, 2010.

\section{Agradecimentos}

Agradecemos ao sujeito da pesquisa, ao Programa de Pós-graduação em Design da UFSC (PósDesign/UFSC), ao Programa de Pós-Graduação em Engenharia de Produção (PPGEP/UFSC), ao Núcleo de Gestão de Design e Laboratório de Design e Usabilidade (NGD-LDU/UFSC), à Rede de Pesquisa e Desenvolvimento em Tecnologia Assistiva (RPDTA), à Universidade Federal da Paraíba (UFPB), à Coordenação de Aperfeiçoamento de Pessoal de Nível SuperiorBrasil (CAPES)- Código de Financiamento 001, ao Conselho Nacional de Desenvolvimento Científico e Tecnológico (CNPq). 